\title{
Negative ion drift velocity and longitudinal diffusion in mixtures of carbon disulfide and methane.
}

\author{
M.P. Dion* \\ CRESST and NASA/GSFC, Code 661, Greenbelt, MD 20771, USA. \\ Department of Astronomy, University of Maryland, College Park, MD 20742, USA. \\ S. Son \\ CRESST and NASA/GSFC, Code 661, Greenbelt, MD 20771, USA. \\ Department of Physics, University of Maryland, Baltimore County, \\ 1000 Hilltop Circle, Baltimore, MD 21250, USA. \\ S.D. Hunter and G.A. de Nolfo \\ NASA Goddard Space Flight Center, Greenbelt, MD 20771, USA.
}

(Dated: April 15, 2011)

\begin{abstract}
Negative ion drift velocity and longitudinal diffusion has been measured for gas mixtures of carbon disulfide $\left(\mathrm{CS}_{2}\right)$ and methane $\left(\mathrm{CH}_{4}\right)$. Measurements were made as a function of total pressure, $\mathrm{CS}_{2}$ partial pressure and electric field. Constant mobility and thermal-limit longitudinal diffusion is observed for all gas mixtures tested. Gas gain for some of the mixtures is also included.
\end{abstract}

*Corresponding author: mpdion@umd.edu 